\title{
Manejo de la Adolescente Embarazada
}

\author{
ASPECTOS MEDICOS
}

\author{
Dr. Alberto Duarte Contreras*
}

El concepto de adolescencia para manejo de problemas obstétricos cobija únicamente a menores de 18 años.

Desde los estudios de Harris en 1922 el embarazo en adolescentes se considera como de alto riesgo y así algunas instituciones atienden a todas las gestantes menores de 18 años en Servicios de Alto Riesgo. Sin embargo, el Proyecto Rochester para la atención obstétrica de las adolescentes, publicado en 1978 no encontró incremento importante de factores de riesgo en las jóvenes de 16 y 17 años. Ya nosotros habíamos llegado a la misma conclusión en un estudio que publicáramos en 1975. En Suecia la atención obstétrica a pacientes de 16 y 17 años se deja al cuidado de los Servicios para adultas sin que se hayan observado diferencias significativas estadísticamente en lo relacionado a complicaciones propias del embarazo y del producto en gestantes adolescentes.

Nosotros, con la experiencia que nos da el manejo de más de diez mil adolescentes embarazadas atendidas en el Hospital San Juan de Dios, de Cúcuta, y en nuestra clientela particular, pertenecientes a todas las capas socioeconómicas, compartimos este concepto y las consideramos para su atención de la siguiente manera: (5)

1. Embarazadas juveniles, a las menores de 14 años.

2. Embarazadas adolescentes, a las de 14 y 15 años.

3. Embarazadas adultas, a las de 16 o más años.

Miembro de Número de la Academia Nacional de Medicina.
En nuestra experiencia las menores de 16 años se deben tratar integralmente como pacientes de alto riesgo, en tanto que las de 16 y 17 años, salvo raras excepciones, por tener un comportamiento obstétrico similar a las de mayor edad tanto en el embarazo como en el parto y en el puerperio hace que las homologuemos para las conductas de atención obstétrica al grupo de las mujeres adultas (2).

Un hecho de importancia para la atención de estas pequeñas pacientes estriba en el estado civil a que pertenezcan. Hasta hace poco se dividían en dos grupos, el de casadas y el de solteras. En la actualidad, desde el punto de vista jurídico, en nuestro país no existe el grupo de casadas puesto que ni la Iglesia ni el Estado unen en matrimonio a menores de 18 años. Ello hace que en lo sucesivo estudiemos un grupo que tiene relación estable y otro que carece de relación estable.

Aquellas que se unen en pareja, forman un hogar y dependen, por lo menos en apariencia, de un varón, constituyen el grupo de relación estable, grupo integrado por $20 \%$ de menores de 16 años y $55 \%$ de adolescentes de 16 y 17 años. Aquellas que no hacen pareja y que permanecen a merced de la aceptación o del rechazo de padres y familiares, se engloban en el grupo de las que carecen de relación estable y constituyen el grupo más numeroso.

¿Qué conducta seguir una vez hecho el diagnóstico de embarazo en una adolescente?

Si pertenece al grupo de las que tienen relación estable, el impacto emocional existe pero no tan crítico pues la mayoría al constituirse en pareja desean el hijo: la noticia del embarazo no las toma por sorpresa ni las lleva a crisis de ansiedad. Con to- 
do, no podemos afirmar que carezcan de problemas pues por falta de un vínculo legal que sostenga la unión, se presenta incertidumbre sobre la estabilidad de la pareja a más de natural zozobra que proviene de los insuficientes recursos económicos que van a ser más golpeados. A estas jóvenes se les debe seguir de cerca y en caso de necesidad, v.g. cuando el varón eludiendo responsabilidad, las abandona, deben recibir ayuda sicológica especial.

A todas las adolescentes embarazadas se les debe ilustrar sobre las complicaciones que se pueden presentar durante la gestación, los cuidados que debe tener, el control prenatal y la necesidad de buscar el servicio hospitalario, para la atención del parto.

Cuando la adolescente pertenece al grupo de carentes de relación estable, debemos volcar en ella toda nuestra atención: constituye el verdadero grupo problema.

Debemos entregarnos a ellas con tiempo, con amabilidad, con comprensión y con conocimiento de materia y de causa.

Esta adolescente al saber que va a ser madre de un hijo, la mayoría de las veces no deseado y aún de padre desconocido, inicia de manera explosiva una crisis emocional que se manifiesta por llanto, temblor y ansiedad; lo niega todo, quiere esconderse o huir despavorida especialmente cuando está en presencia de sus padres. Debemos entonces comprenderla y calmarla para frenar su emotividad. Indagaremos luego con suma cautela sobre la composición familiar y detalles de su vida afectiva, social y escolar para prestarle toda la colaboración requerida con el fin de que pueda informar a sus padres, si es que no están presentes y de ser posible al padre de su futuro hijo sobre el embarazo que cursa. A ellos se les debe enseñar el comportamiento que deben seguir ante un hecho cumplido. Esta ayuda se puede prestar personalmente o a través del Servicio Social o del Voluntariado Hospitalario.

No se puede permitir un enfrentamiento verbal ni menos de hecho entre la adolescente y su familia. El razonamiento, el buen criterio y el consejo del médico son un factor decisivo para impedir uniones a la fuerza.

Desde el primer momento se le debe dar un soporte sicológico insistiéndole en que por ningún motivo debe abandonar el hogar ni su trabajo y se le ayudará a resolver el problema de la deserción es- colar, la que debe ser transitoria. El médico tratante debe saber que esta consulta ha de ser algo más que una simple exploración física. Debe servir de orientación sicológica y familiar y por ello se le debe dar todo el tiempo que sea necesario.

A la familia se le demostrará la incapacidad física en que se encuentra la jovencita para responder por su sustento y el de su futuro hijo. Es indispensable insistir que la hija ahora más que nunca necesita cariño y comprensión, los que muchas veces se le han negado. Es el momento más propicio para recalcar a la familia y a la hija la necesidad física y moral de conservar su embarazo y de no exponerse al grave riesgo que le implicaría someterse a un aborto provocado que comprometería su integridad física y tatuaría con estigma subienestar moral. $\mathrm{La}$ adolescente embarazada necesita entonces se le dé apoyo excepcional, manifestaciones de calor y de cariño hogareño a más de una completa orientación médica y social. A este respecto es indispensable poner en juego todo el poder de persuasión y de convencimiento de que sea capaz el médico tratante. En oportunidades la adolescente es lanzada del hogar por padres intransigentes; se le puede entonces ayudar a instalar en un lugar con ambiente favorable y apacible como el de un albergue o casa para madres solteras. Esta colaboración se hace indispensable cuando la joven carece de padres y los familiares no le prestan la menor colaboración. Es entonces cuando juega un especial papel el servicio social hospitalario o las instituciones de damas voluntarias o de la caridad.

El soporte sicológico para este grupo debe ser constante e ininterrumpido durante todo el embarazo y aún después del parto. Esta labor, en cuanto sea posible, debe llevarse a la familia y a los responsables de la educación de la joven, vale decir a sus maestros.

Queremos llamar la atención en el hecho de que el impacto y desajuste emocional ante un embarazo no deseado es tan violento en las adolescentes de menor edad como en las de 16 y 17 años, así pertenezcan al grupo de las que tienen o no tienen relación estable $(4)$.

Se debe insistir hasta la saciedad en la importancia de la consulta prenatal, la que debe ser especializada y para ello es indispensable que se tomen todas las medidas necesarias estableciendo servicios de consulta externa obstétrica para las adolescentes de menor edad en los hospitales y servicios de atención médica. 
Las consultas que se hagan en el período prenatal se deben iniciar con un enfoque sicológico antes de pasar a la parte obstétrica. En la primera consulta se practicará un examen general, en el cual se prestará sumo cuidado al explorar el estado nutricional y luego se ordenarán los exámenes paraclínicos de rigor. Como en toda embarazada se tomará el dato de peso, tensión arterial y altura uterina.

En todas las consultas es indispensable practicar examen de orina y poner especial cuidado en la investigación de peso, tensión arterial y presencia de edema en miembros inferiores (7).

\section{DESNUTRICION}

El embarazo y la lactancia en adolescentes plantean problemas especiales de nutrición que requieren un enfoque multidisciplinario. $\mathrm{Al}$ examinar a nuestras adolescentes embarazadas encontramos un alto porcentaje con problemas de nutrición el que no estamos en capacidad de calcular; es más acentuado en el grupo de menor edad y en aquellas que han abandonado el hogar y el trabajo. Estas adolescentes son por lo general jovencitas que provienen de hogares pobres, o que pueden tener caprichos alimenticios, o que siguen hábitos dietéticos de moda para adelgazar, o que han padecido recientemente infecciones agudas, o portadoras de enfermedades crónicas, o entregadas al vicio del alcohol, de la droga o del tabaco, o que no tienen donde dormir ni reposar y deambulan por las calles día y noche, algunas de ellas entregadas a la "promiscuidad lucrativa", o muchachas recién llegadas del campo, sin trabajo, poliparasitadas, acosadas por traumas impactantes sicológicos producidos por la inseguridad rural o por la reciente pérdida alevosa de sus padres o parientes, carentes todas de una dieta suficiente, hechos que requieren un enfoque multidisciplinario tanto para el diagnóstico como para el tratamiento de la falla nutricional.

Esta falta de reservas y carencia de ingestión de nutrientes en las adolescentes retrasa el crecimiento propio de la joven lo mismo que el desarrollo y la viabilidad del feto y luego durante el período de lactancia aumenta la morbimortalidad neonatal. La adolescente que se embaraza a muy temprana edad, cuando se encuentra en plena etapa de crecimiento, tiene proporcionalmente necesidades mayores nutritivas y calóricas que una mujer adulta puesto que hay en ella una superposición de necesidades nutricias: las de su crecimiento físico, las de su maduración sexual, las propias del desarrollo fetal más otros factores no bien conocidos sobre los cuales se encuentran aún muchos vacíos. El aporte ideal que debiera recibir se aprecia en $1.3 \mathrm{~g} / \mathrm{K} /$ día (6).

Las adolescentes embarazadas en su mayoría pertenecen a los estratos económicamente más necesitados y por ello su alimentación es altamente deficiente no sólo en cantidad sino en sustancias proteicas, hierro, calcio y vitaminas hecho que se puede observar por la clínica y demostrar por el laboratorio (bajos niveles de proteínas y de hemoglobina, a más del poliparasitismo intestinal y hemático). Todo ésto hace que disminuyan el crecimiento y la maduración de la adolescente como también el desarrollo fetal y si el estado de desnutrición se extiende hasta el período de la lactancia, aumenta la mortalidad neonatal.

No hay duda de que la desnutrición juega un papel importante en la evolución y pronóstico de la toxemia y es causante del parto pretérmino, del bajo peso de los productos y de la mortalidad perinatal. También es posible que haya un aumento de anomalías congénitas en recién nacidos de madres desnutridas. Los embarazos repetidos y a breves intervalos agravan aún más la carencia nutricional y aumentan la posibilidad de complicaciones maternas e infantiles perinatales.

Ello hace que en todo plan de atención prenatal en adolescentes embarazadas las medidas nutritivas constituyan un componente integral. Se ha de mostrado que cuando se presenta oportuna vigilancia médica y se administra un adecuado aporte nutricional más soporte sicológico, la proporción de las complicaciones que se puedan presentar se acerca mucho al que corresponde a una población obstétrica de mayor edad.

A todas estas jóvenes se les debe desparasitar y tratar las enfermedades intercurrentes antes de iniciar el tratamiento nutricional. Y es aquí donde empieza el problema dados los exiguos recursos familiares y estatales. Se debe entonces procurar en cuanto sea posible hospitalizar a estas pequeñas pacientes para buscar la manera de administrarles por el mayor tiempo posible una alimentación hiperproteica y balanceada que satisfaga en algo sus necesidades. Es indispensable que las dietas se adapten a los hábitos alimenticios de la joven y a las necesidades emocionales y sociales; por ello deben ser dietas individuales. La hospitalización es difícil en hospitales regionales o universitarios, pero éstos pueden remitir a centros de salud o a hospitales satélites, pequeños, de poblaciones vecinas, por lo general subutilizados. 
El valor biológico de las proteínas se rige por los componentes relativos de aminoácidos. En las embarazadas las proteínas animales, leche, huevos, son mejor fuente nutricional que las vegetales. Además, como no todas las proteínas poseen los aminoácidos en proporción adecuada, se aconseja ingerirlas en exceso; desafortunadamente sus costos sobrepasan los reducidos presupuestos hogareños y aún hospitalarios.

Todo tratamiento encaminado a mejorar la desnutrición debe tener en cuenta una serie de trastornos previos o actuales con ingerencia sobre el estado nutricional tales como los embarazos anteriores, las enfermedades infecciosas agudas o crónicas en evolución, trastornos gastrointestinales, parasitosis, problemas de mala absorción, hábitos alimenticios, dietas de adelgazamiento. Por ello, para el estudio y tratamiento de la desnutrición en las adolescentes embarazadas es básica la historia pormenorizada de la joven que incluya el conocimiento de los antecedentes familiares y personales. El alcohol, el cigarrillo y las drogas tienen una particular importancia por la nefasta acción contra el feto,sobre todo al principio del embarazo. Los problemas emocionales no se pueden pasar inadvertidos. Es importante tener en cuenta que la adolescente puede estar empleando la indisciplina nutritiva como arma contra ella misma o contra el desarrollo del feto.

La División Materno Infantil del Ministerio de Salud y nuestros hospitales, a pesar de conocer el incontrolable aumento del embarazo en adolescentes y la infinidad de problemas asistenciales y sociales que de él surgen, no ha podido adelantar programas especiales de diagnóstico y atención a este déficit nutricional dado los presupuestos siempre deficitarios a que los tienen sometidos.

Hoy, con subsidio del Estado, tenemos en nuestro país un complemento dietético de gran utilidad para las adolescentes embarazadas de bajos recursos económicos, que brindan los requerimientos calóricos y nutritivos necesarios: la Bienestarina. Es una harina de color ligeramente amarillo, textura fina, sabor y olor característicos dela harinade maíz. Se elabora con una mezcla de harina de maíz gelatinizada, harina de trigo, torta de soya, leche en polvo, metionina, vitaminas y minerales. nan:

Cada 100 gramos de bienestarina proporcio-

$\begin{array}{lclr}\text { Calorías } & 319 & \text { Calcio } & 512 \mathrm{mg} . \\ \text { Agua } & 9.7 & \text { Fósforo } & 766 \mathrm{mg} . \\ \text { Proteínas } & 26 \mathrm{~g} . & \text { Hierro } & 14.1 \mathrm{mg} .\end{array}$

$\begin{array}{lrlr}\text { Grasas } & 1.4 \mathrm{~g} . & \text { Vitamina A } & 2000 \mathrm{U} . \mathrm{I} . \\ \text { Carbohidratos } & 58.2 \mathrm{~g} . & \text { Tiamina } & 1.99 \mathrm{mg} . \\ \text { Fibra } & 1.4 \mathrm{~g} . & \text { Riboflavina } & 0.5 \mathrm{mg} . \\ \text { Cenizas } & 3.3 \mathrm{~g} . & \text { Niacina } & 9.7 \mathrm{mg} . \\ & & \text { Vitamina C. } & 27 \mathrm{mg} .\end{array}$

Es una harina de excepcional valor nutricional y con ella se pueden preparar sorbetes de frutas, sopas con vegetales, coladas de dulce o de sal, arepas, purés, etc.

A más de la administración de proteínas se debe administrar aporte calórico, calcio e hierro.

\section{APORTE CALORICO}

El embarazo representa un período de rápido crecimiento y desarrollo de tejidos maternos y fetales y por ello la adolescente que está en pleno creci. miento tendrá mayores necesidades calóricas que la mujer adulta.

La exigencia calórica máxima guarda paralelismo con la curva de crecimiento y dado que cada adolescente tiene su cuadro peculiar de desarrollo, las raciones calóricas deben satisfacer necesidades individuales y el cálculo de esta necesidad calórica individual debe fundarse en el desarrollo fisiológico y no en la edad cronológica.

Se ha calculado que el ingreso calórico para jóvenes entre 12 y 17 años, en plena actividad y desarrollo, debe estar alrededor de 2.100 calorías. Las diferentes dietas seguidas por muchas adolescentes embarazadas para guardar la línea o para ocultar su embarazo produce muy bajos ingresos de proteínas y de hierro al igual que de calorías. Una buena ganancia de peso de 10 a 12.5 kilos durante el embarazo, se considera el mejor índice clínico de ingreso calórico normal.

Algunos autores sostienen que no se debe restringir el aporte calórico en las adolescentes obesas. Nosotros creemos que se debe restringir el aporte en estas pacientes so pena de agregar un factor más de riesgo cual sería el aumento exagerado del peso de la madre y del producto que bien podría constituir luego una distocia.

\section{CALCIO}

Durante el embarazo se necesita una retención adicional de calcio principalmente para mineralizar el esqueleto del feto. El aporte mayor de calcio se requiere durante el tercer trimestre. La necesidad fetal de calcio puede ser satisfecha por las reservas que tenga la madre, pero estas adolescentes emba- 
razadas que están en crecimiento y que por lo general son mal nutridas no gozan de reservas suficientes para atender esta doble necesidad.

En las adolescentes durante la gestación se encuentran adaptaciones metabólicas que originan mecanismos especiales para absorber el calcio con celeridad en el aparato gastrointestinal y conservarlo de la manera más eficaz. Pero ante una alimentación deficiente, mal se pueden mantener las reservas necesarias para el doble crecimiento, el de sí misma y el de su producto. Casos habrá en los que el aporte alimenticio sea tan exiguo y la reserva materna en calcio sea tan baja, que se pueda llegar a la osteomalacia materna y al raquitismo fetal. Además un ingreso inadecuado de calcio durante el embarazo puede perturbar más tarde la lactancia.

\section{HIERRO}

La anemia es muy frecuente en la adolescente embarazada cualquiera sea la altitud a que se encuentre. Para algunos autores la anemia es patrimonio del embarazo a esta edad. Nosotros no la consideramos como tal, sino que la catalogamos como resultado lógico de la desnutrición y del poliparasitismo tan comunes en las clases menos favorecidas. La que encontramos es una anemia generalrnente del tipo ferropénico (1).

La mayor parte de la demanda de hierro ocurre durante la segunda mitad de la gestación. La necesidad total de hierro debe satisfacer el aumento de la eritropoyesis de una mujer que está en plena fase de desarrollo, el de un feto en evolución, el de la placenta funcionante, el de la futura lactancia más una reserva para eventuales pérdidas hemátiças como la que sucede durante el parto. Afortunadamente la adolescente, y más durante el embarazo, tiene mayor poder de absorción de hierro que la mujer adulta. Se calcula que la adolescente embarazada absorbe normalmente el 10\% del hierro dietético, proporción que aumenta hasta el $25 \%$ durante el último trimestre de la gestación.

Un alto número de adolescentes inicia su embarazo con notable disminución de la reserva férrica, hierro que no alcanzan a absorber de la escasa y deficiente dieta recibida; por ello se hace necesaria la administración de suplementos férricos a razón de 100 a $200 \mathrm{mg}$. diarios dados en forma de sulfato, fumarato o gluconato ferroso puro o asociado a sustancias que incrementan la absorción intestinal tales como el ácido fólico y el ácido ascórbico.

La deficiencia férrica se hace más notoria en las adolescentes que tienen repetidas y cercanas gestaciones, hecho que sugiere la presencia de un déficit acumulativo de hierro.
Las complicaciones hemorrágicas del embarazo como el aborto, el extrauterino roto, la mola, la placenta previa, el desprendimiento prematuro de placenta, las hemorragias postparto requieren atención especial como también reposición del volumen de sangre perdida administrando sangre total o glóbulos empaquetados.

\section{REPOSO}

El reposo es otro de los métodos especiales usados en adolescentes embarazadas que se encuentren profundamente afectadas desde el punto de vista sicológico. La adolescente vive en continua actividad física y emocional; con el reposo se tranquili$\mathrm{za}$, entra en relajación corporal placentera, disminuye las necesidades calóricas y proporciona tiempo oportuno para adelantar el tratamiento sicoterápico. La cura de reposo se debe realizar en una institución hospitalaria pues sólo así estamos seguros de que se cumple a cabalidad y podremos prestar atención de alto riesgo a las menores de 16 años, servicio que por demás requiere la presencia de personal bien entrenado en el manejo de estas pequeñas pacientes. La hospitalización de las menores de 16 años, de ser posible, se hace en una sala especial para adolescentes embarazadas donde pueda permanecer separada de las mujeres de mayor edad. Además es de suma importancia distribuir el tiempo a estas niñas de manera racional entre reposo, sueño y recreación (3).

El reposo absoluto en cama se aconseja en la amenaza de aborto, amenaza de parto prematuro, ruptura prematura de membranas, en crisis emocionales y primordialmente en la toxemia.

La toxemia es una complicación frecuente que hemos constatado en el $38.53 \%$ de las menores de 16 años. En la apreciación de diferentes autores se encuentra con una frecuencia cinco veces más que en las de mayor edad. Tal vez la frecuencia obedezca a la desnutrición de estas pequeñas pacientes. A las adolescentes se les debe hospitalizar a la menor sospecha de toxemia: una pequeña elevación tensional, inicio de edemas, proteinuria, aumento de peso por encima de lo normal, ya que con suma frecuencia nos sorprenden toxemias severas y aún eclampsia en jovencitas que presentaban una muy discreta sintomatología. Su tratamiento se hace primordialmente con reposo absoluto en cama seguido de dieta hiperproteica y sedación de su estado emocional con sicoterapia y a veces con administración de diazepan. Con estas medidas la paciente regresa fácilmente a la normalidad.

E1 aborto provocado exige hospitalización, tratamiento de la infección, evacuación uterina y orientación sicológica. Por su importancia me quiero referir a la evacuación uterina. Debe realizarse 
siempre bajo anestesia general y ha de practicarse con sumo cuidado evitando maniobras bruscas que puedan ocasionar perforación uterina. Es preferible emplear cucharillas cortantes las que se toman entre los dedos índice y pulgar y las manipulaciones han de ejecutarse solamente con la fuerza que proporcionan estos dedos. En esta forma evitaremos extraer porciones de miometrio lo que bien podría ocasionar sinequias causantes de amenorrea y de esterilidad de no ser diagnosticadas y tratadas adecuadamente. Las maniobras bruscas en la vecindad del orificio interno pueden ocasionar una incompetencia cervical causante de abortos repetidos. Insistimos que estos legrados deben ser practicados por personal entrenado. En estos casos se impone una fuerte dosis de sicoterapia más instrucción de métodos anticonceptivos. No puede haber barrera alguna que impida a una adolescente obtener la suficiente información sobre la planificación familiar.

\section{OTROS CUIDADOS}

Mientras transcurre el embarazo, durante las consultas prenatales y mejor aún en charlas programadas se le debe enseñar a la adolescente, de acuerdo con sus conocimientos y sus capacidades lo que son el embarazo y el paso del producto por el canal vaginal; qué es la operación cesárea, la que sólo se necesita en el 5 ó $6 \%$ de las parturientas adoiescentes; qué es la episiotomía y por qué en oportunidades se emplean las espátulas. Se les debe informar con detalle sobre la lactancia, el manejo del recién nacido, la crianza del niño a más de la necesidad de la planificación familiar. Hay una serie de enseñanzas y medidas de índole social y económica que pudieran darse en centros especializados en capacitación para la vida como el Sena y el Instituto de Bienestar Familiar.

Cuando la gestante tiene un aceptable cuidado prenatal y recibe oportuno tratamiento a las complicaciones de su embarazo, disminuye el número de partos pretérmino, de productos de bajo peso y de mortalidad perinatal. Desafortunadamente esta atención no se cumple entre nosotros. Si bien el número de embarazos en el grupo de 16 y 17 años va disminuyendo, se nota un sensible aumento en las de menor edad y la asistencia de este grupo a la consulta prenatal es casi nula, lo que hace que más del $80 \%$ de estas jóvenes lleguen al hospital sin control prenatal, en trabajo de parto, con embarazos que escasamente llegan a las 35 a 36 semanas, en precarias condiciones generales, con complicaciones propias del tercer trimestre del embarazo o del trabajo de parto. A ellas se les debe practicar de inmediato no sólo el examen obstétrico sino el examen general clínico y paraclínico, cuando de tiempo; se hará un cuidadoso seguimiento del trabajo y de la atención del parto.

En ausencia de complicaciones obstétricas, se dejará que el parto siga un curso normal sin acelerarlo con ocitocina. El poder contráctil de la fibra muscular uterina de la adolescente desarrolla un dinamismo que no necesita ayuda alguna so pena de agotarlo y producir distocia contráctil. Se pueden aplicar espátulas de desprendimiento, previa la episiotomía. La episiorrafia debe ser muy cuidadosa, conservando minuciosamente la arquitectura anatómica. La cesárea se verifica sólo por indicación obstétrica y nunca para acelerar el trabajo que de por sí es corto. Las normas obstétricas y no la edad de la paciente son los indicadores de la conducta que se debe seguir en la atención del parto.

Los recién nacidos deben ser entregados de inmediato a la atención del pediatra.

Es de gran utilidad entrevistar posteriormente a los padres de la joven con el fin de insistirles en los cuidados y el cariño que deben prodigar a la hija y al recién nacido.

En resumen, el cuidado que se debe prestar a la adolescente embarazada consiste primordialmente en la atención de su crisis emocional y luego mejorar su estado nutricional, tratar las diversas complicaciones obstétricas o intercurrentes, vigilar solícitamente el parto por personal idóneo, enseñar la planificación familiar, orientar su sexualidad y prepararla para el cuidado y educación del hijo sin olvidar las pautas que se deben dar a la familia. Las menores de 16 años deben ser atendidas en Servicio de Alto Riesgo.

\section{BIBLIOGRAFIA}

1. DUARTE-CONTRERAS, A. Embarazo en adolescentes de 11 a 15 años. Rev. Colomb. Obstet. Ginecol. 14: 259, 1973.

2. DUARTE-CONTRERAS, A. ¿El embarazo en adolescentes es siempre de alto riesgo? Rev. Colomb. Obstet. Ginecol. 26: $385,1975$.

3. DUARTE-CONTRERAS, A. Bases para la atención ginecológica especializada en niñas adolescentes. Rev. Colomb. Obstet. Ginecol. 32: 299, 1981.

4. DUARTE-CONTRERAS, A.; BARreto, L. A. Factores de- terminantes del embarazo en adolescentes solteras. Rev. Colomb. Obstet. Ginecol. 36: 291, 1985.

5. DUARTE-CONTRERAS, A. Adolescencia y embarazo. En: Ginecología de la niña y la adolescente. Ed. Salvat, en prensa, 1988.

6. GREENHILL, J. P.; FRIEDMAN, E. A. Obstetricia. Ed. Interamericana, 1980.

7. SIMPOSIO. Problemas de Reproducción en Adolescentes. Clínicas Obstétricas y Ginecológicas. 325-488, junio-1971. 